\title{
Etude biochimique de l'albinisme chez les Mammifères et chez l'Homme
}

\author{
C. VOULOT \\ Laboratoire de Biologie, I.N.S.A., 20, avenue Albert-Einstein \\ 69621 Villeurbanne Cedex
}

\begin{abstract}
Résumé
Divers mécanismes biochimiques sont susceptibles d'expliquer l'expression de l'albinisme vrai des Mammifères au Locus $C$ qui, contrôlant directement la synthèse de tout pigment mélanique, a un effet qui doit être lié à l'activité des tyrosinases.

L'hypothèse d'un arrêt de la synthèse des tyrosinases paraît évidente dans certains cas, par contre cette hypothèse doit être rejetée chaque fois que des tyrosinases actives in vitro peuvent être extraites des organes pigmentaires des individus albinos.

L'inhibition des tyrosinases a été fréquemment décrite au niveau de la peau ou des bulbes pileux d'animaux pigmentés. Deux mécanismes sont connus : l'inhibition directe de l'enzyme, ou son inhibition compétitive limitant la quantité de substrat disponible. Malheureusement, nous ne disposons que de deux cas d'études biochimiques montrant une inhibition des tyrosinases dans des cas d'albinisme. Un cas concerne le mutant albinique du mélanome S91 de la Souris, l'autre l'albinos oculocutané humain. Il est frappant de constater que, dans ces deux cas on voit coexister un type d'albinisme par absence d'enzyme et un autre type par inhibition enzymatique. C'est ainsi que, si l'albinisme de type A pour le mélanome S91 de la Souris s'explique par l'absence de tyrosinase, l'albinisme de type B implique l'inhibition de cette dernière.

Nous devons constater qu'actuellement, l'insuffisance des résultats expérimentaux empêche la vérification des théories proposées. Les Rongeurs chez lequels la mutation albinique est fréquente et la génétique pigmentaire bien connue devraient constituer un matériel de choix, or même chez ces animaux on ne peut établir avec certitude si l'albinisme s'explique par absence ou inhibition des tyrosinases. En effet, dans la plupart des cas, l'étude électrophorétique montre l'absence de ces enzymes alors que les techniques de marquage radioactif de la tyrosine établissent l'activité enzymatique correspondante.
\end{abstract}

Chez les Mammifères l'albinisme est contrôlé génétiquement par le locus $C$. On connaît la série allélique correspondante, caractérisée par une réduction progressive de la pigmentation mélanique.

L'allèle dominant $C$ permet l'expression inaltérée des divers allèles liés aux autres loci. Au contraire l'ultime forme récessive $c$, à l'état homozygote inhibe toute expression pigmentaire. On obtient alors la dépigmentation simultanée et totale de la peau, des poils et de l'œil ; c'est le phénotype albinos classique bien connu chez la plupart des Rongeurs. 
Nous remarquerons que la série allélique du locus $C$ comporte divers intermédiaires entre les deux extrêmes que nous venons d'évoquer, $C$ et $c$. On assiste à une réduction progressive de la pigmentation, mais les deux types de mélanine, eumélanine et phaeomélanine peuvent être diversement affectés.

Chez les allèles «Chinchilla», le pigment jaune (phaeomélanine) est réduit sélectivement.

Pour l'allèle «Himalaya», seule subsiste une pigmentation eumélanique limitée aux extrémités (acromélanisme).

Quelle interprétation la biochimie est-elle actuellement en mesure de fournir au modèle proposé par la génétique?

Dans le cas de la série albinique une relation s'impose, puisque le locus $C$ contrôle directement la synthèse mélanique, à la différence de loci dont les effets correspondent à des modifications des mélanocytes, des mélanosomes ou de l'environnement cellulaire (SEARLE, 1967).

Un contrôle enzymatique s'exerce à deux niveaux sur les voies métaboliques des mélanines mammaliennes.

- Les tyrosinases*, enzymes clefs qui en catalysant l'oxydation initiale du substrat commandent l'ensemble du processus.

- D'autres enzymes contrôlent la mobilisation de la cystéine nécessaire à la synthèse des seules phaeomélanines (Prota \& Searle, 1978).

Il est assez logique de lier l'effet du locus $C$ à la seule tyrosinase puisque le gène $c$ à l'état homozygote inhibe toute synthèse pigmentaire ou phaeomélanique. Cette idée est renforcée par le remarquable modèle expérimental que nous propose l'allèle Himalaya chez le Lapin. Chez ces animaux la pigmentation normalement limitée aux extrémités peut s'étendre sous l'effet d'un abaissement de la température ambiante ou au contraire se réduire sous l'effet d'une élévation de température (Robinson, 1958). Une telle thermodépendance est liée à la thermolabilité de la tyrosinase caractéristique de laallèle $C^{\mathrm{h}}$.

On peut concevoir l'action du locus $C$ sur les tyrosinases à deux niveaux :

- au niveau de la synthèse de l'enzyme qui est modifiée (tyrosinase thermolabile pour $C^{\mathrm{h}}$ ) ou supprimée $(c)$;

- au niveau du fonctionnement de l'enzyme qui peut être inhibée par un agent externe.

Seul le premier modèle fut initialement envisagé et l'on considéra que le locus $C$ constituait un locus structural pour les tyrosinases (Foster, 1965). Pourtant des résultats biochimiques récents permettent de montrer l'existence de tyrosinases actives chez des individus albinos ( $c$ homozygote) et nous obligent à considérer avec attention les mécanismes de l'inhibition des tyrosinases.

* Nous parlerons de «tyrosinases », car dans la plupart des matériels mélaniques on a pu mettre en évidence trois isoenzymes distinctes; $T_{3}, T_{2}, T_{3} . T_{1}$ et $T_{2}$ sont solubles, $T_{3}$ est au contraire lié aux mélanosomes et ne peut être libéré que par l'action de détergents. 


\section{Des tyrosinases actives sont-elles présentes chez les Rongeurs albinos ?}

Les diverses isoenzymes des tyrosinases mammaliennes furent décrites après une séparation électrophorétique associée à une révélation sur le gel par réaction de DOPA. Le matériel non pathologique le plus couramment étudié fut le bulbe pileux des Rongeurs. Pour toutes les souches correspondant à des phénotypes pigmentés, il est possible de visualiser sur gel des bandes mélaniques qui trahissent la présence des diverses enzymes tyrosinases. Pour toutes les souches albinos au contraire, les gels restent clairs et l'observateur doit conclure à l'absence des tyrosinases. De tels résultats sont rapportés par HolsTein et al. à la fois chez la Souris (1967) où un grand nombre de souches furent étudiées, et chez quatre autres espèces de Rongeurs ; Lapin, Rat, Hamster, Gerboise (1971).

Pour un autre type de matériel pigmentaire, les organes mélaniques de l'œil (choroïde, iris, processus ciliaires), l'étude électrophorétique aboutit aux mêmes conclusions. Les tyrosinases sont présentes chez les animaux pigmentés, et absentes chez les albinos. Ces résultats ont été vérifiés chez la Souris, le Rat, le Lapin et le Hamster (Voulot \& Laviolette, 1976 ; Voulot \& Ortonne, 1979). On doit cependant signaler une exception à cette absence systématique des tyrosinases sur les préparations électrophorétiques de matériel albinos. Il s'agit du mutant albinique (type B) obtenu par ChIan \& Wilgram (1967) à partir du mélanome S 91 de la Souris. Ces auteurs ont pu isoler à partir de ce matériel les trois isoenzymes $T_{1}, T_{2}$ et $T_{3}$ des tyrosinases. La forme $T_{2}$ purifiée fut mise en évidence sur gel de polyacrylamide et sa mobilité est légèrement inférieure à l'isoenzyme correspondante, obtenue à partir du matériel pigmenté.

Si par contre on utilise pour caractériser les tyrosinases, non plus la séparation électrophorétique mais un marqueur radioactif (Pomerantz, 1964), la présence de tyrosinase active peut être démontrée dans la plupart des matériels albiniques testés. La méthode proposée par PoMERANZ consiste rappelons-le à caractériser les tyrosinases par leur activité sur la tyrosine; on mesure la radioactivité de la DOPA obtenue à partir du substrat marqué (tyrosine).

L'activité de la tyrosinase a ainsi été démontrée dans des extraits cutanés de Rat albinos par Gaudin \& FELlman (1972). Des résultats similaires ont été publiés par Pomeranz \& Li (1974) pour la Souris et le Hamster. Hearing enfin a publié en 1973 une étude comparative de l'activité des tyrosinases oculaires chez les Souris albinos et pigmentées. Cet auteur mesure la radioactivité de la mélanine obtenue par incubation d'extraits tissulaires ou de fractions mélanosomiales dans de la tyrosine marquée. Le matériel obtenu à partir des animaux albinos montre une activité de la tyrosinase inférieure à celle des animaux pigmentés, mais néanmoins notable.

Nous ajouterons enfin à notre exposé d'autres cas dans lesquels l'activité de la tyrosinase est démontrée chez les albinos. Je veux parler de l'albinisme humain. Kugelman \& Van ScotT (1961) obtinrent la formation de pigment mélanique en incubant dans de la tyrosine ou de la DOPA des bulbes pileux de sujets humains albinos. Des travaux ultérieurs permirent de décrire chez l'Homme une forme d'albinisme où les bulbes pileux produisent de la mélanine lorsqu'on les incube dans de la tyrosine (albinisme tyrosinase + ) et une autre forme où aucune mélanisation des bulbes ne peut être obtenue (WITKOP et al., 1973). 


\section{L'albinisme correspond-il à une inhibition des tyrosinases ?}

L'existence d'inhibiteurs chimiques de la mélanogénèse a été signalée depuis longtemps dans les tissus où la mélanogénèse est observée : peau, œil, mélanome. On peut regrouper ces inhibiteurs en deux grandes catégories :

- les inhibiteurs de faibles poids moléculaires, qui généralement possèdent un groupement sulfhydryle (SАTOH \& Mishima, 1967 ; SeiJi et al., 1967); 1970 a-b).

- les inhibiteurs de nature polypeptidique ou protéique (FLAWN \& WILDE,

Dans la plupart des cas on est en présence d'une inhibition compétitive des tyrosinases. Généralement ces études ne s'appliquent pas directement à un cas d'albinisme. Il en va autrement du travail de ChIan \& Wilgram (1967). Ces auteurs décrivent un inhibiteur de nature protéique extrait de mélanosomes (S 91 de la Souris) présentant des mutants albiniques. Or, il se trouve qu'un type de tumeur mutante ne possède pas de tyrosinase (albinisme de type A), alors que l'autre type au contraire possède des tyrosinases actives in vitro pouvant être extraites et purifiées.

L'intérêt des travaux de ChIAN \& Wilgram vient de ce que ces auteurs ont pu proposer un mécanisme de l'inhibition des tyrosinases induisant l'albinisme.

L'inhibiteur tumoral agit sur les tyrosinases solubles qu'elles viennent de mélanome pigmenté ou de la tumeur albinique de type B. Par contre s'il s'agit de la tyrosinase liée aux mélanosomes $\left(\mathrm{T}_{3}\right)$ l'inhibition est observable dans le cas des tumeurs albiniques de type $B$ mais nulle pour le matériel provenant des tumeurs pigmentées. CHIAN \& Wilgram à partir de ces observations ont supposé que l'inhibition générale des tyrosinases libres cesse lorsque l'enzyme se trouve intégrée dans la matrice protéique du mélanosome $\left(\mathrm{T}_{3}\right)$. L'albinisme de type $\mathrm{B}$ correspondrait à une persistance anormale de l'inhibition de la tyrosinase $T_{3}$ dans le mélanosome, inhibition qui resterait possible du fait d'une modification de la structure mélanosomiale. De fait, les auteurs ont pu observer en microscopie électronique cette malformation des mélanosomes des tumeurs type B. La portée de la théorie de ChIAN \& WILGRam dépasse largement la seule explication d'un albinisme par inhibition des tyrosinases, car ils proposent en outre un modèle qui rend compte de la localisation de la synthèse mélanique dans les mélanocytes. La mélanine ne se forme en effet que dans les mélanosomes bien que des tyrosinases solubles, actives in vitro puissent être extraites à partir d'autres fractions cellulaires (ribosomes, etc.).

Le travail de Chian \& Wilgram a été confirmé par Seiji et al. (1973) sur le mélanome HaRding-Passey dans la mesure où ces auteurs ont retrouvé sur ce matériel des fractions protéiques inhibant les tyrosinases solubles de la tumeur, mais n'agissant pas sur la tyrosinase particulaire. SEIJI et al. cependant ne disposaient pas avec le mélanome HARDING-PASSEY d'un système analogue aux mutants albinos du S 91.

\section{Conclusions}

A la lumière des résultats dont nous disposons, quelques remarques principales s'imposent : 
1) Deux mécanismes biochimiques de l'albinisme paraissent coexister ; l'absence de tyrosinases actives et l'inhibition de ces dernières. Cette dualité du mécanisme est d'autant plus frappante que dans les cas les mieux étudiés elle peut se manifester pour le même matériel (albinisme de type $A$ et de type $B$ pour les mélanomes $S 91$, albinisme humain tyrosinase négatif ou tyrosinase positif).

2) L'inhibition des tyrosinases induisant l'albinisme peut être envisagée comme une inhibition compétitive limitant le substrat disponible ou au contraire comme une inhibition directe de l'activité de la tyrosinase. La tumeur étudiée par CHIAN \& WiLGRAM correspond à une inhibition directe de la tyrosinase dans le mélanosome. Au contraire, l'albinisme humain de type tyrosinase positif paraît se ramener à une inhibition compétitive puisque l'incubation en présence d'un excès de substrat permet la mélanogénèse.

3) Une inhibition directe de l'activité de la tyrosinase reste compatible avec la conception du locus $C$ comme locus structural pour les tyrosinases. Dans le modèle de Chian et Wilgram l'inhibition de la tyrosinase $T_{3}$ peut être causée par une modification structurale de l'enzyme ne pouvant plus s'organiser dans le mélanosome en une structure régulière protectrice. De la même manière, les lapins de génotype Himalaya synthétisent des tyrosinases modifiées sensibles à l'inhibition thermique. Au contraire, l'albinisme induit par une inhibition compétitive de la tyrosinase paraît incompatible avec la conception du locus $C$ comme locus structural.

Ces quelques idées générales ne doivent pas masquer l'insuffisance persistante des résultats biochimiques concernant l'albinisme mammalien. Le cas des mutants albinos du mélanome $\mathrm{S} 91$ dont l'étude est déjà vieille de 13 ans, constitue à peu près le seul exemple d'une étude biochimique cohérente. Force nous est de constater qu'il n'existe pas d'étude portant sur un matériel albinique non tumoral. Chez les Rongeurs où la mutation albinos est fréquente et génétiquement bien connue, le problème de la présence ou de l'absence des tyrosinases reste irrésolu dans la mesure où la séparation électrophorétique et le «radioassay» de Pomerantz fournissent généralement des résultats contradictoires.

Reçu pour publication en mars 1981.

\section{Summary}

\section{Biochemical study of albinism in Mammals and Man}

Several biochemical processes are involved in the mode of action of alleles at the Albino locus C. Such alleles act directly on melanin synthesis and their influence must be considered as mediated by action on the tyrosinase activity.

The lack of tyrosinase may often explain albinism but evidence against this hypothesis comes from reports that, in vitro, active tyrosinase is extracted from pigmentary organs of albino animals. The tyrosinase inhibition has been described in the skin and hairbulbs of the pigmented animals. Two types of inhibition are reported : direct inhibition of the enzyme or competitive inhibition acting on the substrate availability. Unfortunately evidence of tyrosinase inhibition has been obtained in two cases only with albino material : the non pigmented albino strain of the S 91 Mouse melanoma and the human oculocutaneous albinism. It must be pointed out that in both cases it has been possible to describe a type of albinism induced by lack of tyrosinase and another type induced by tyrosinase inhibition.

For instance with the type B albino mutation of the S91 Mouse melanoma, active tyrosinase is not extractable, in contrast in the type $\mathbf{B}$ albino mutation of the same tumor an active tyrosinase is present and its inhibition demonstrated. 
It must be said that presently the general biochemical investigation of mammalian albinism has not been performed. With Rodents, the best known Mammals from the point of view of albinism and coat colour genetics, electrophoretic separation of tyrosinase shows a lack of enzyme in albinos but the radioassay of labelled tyrosine give often evidence for active albino tyrosinase and enzymatic inhibition.

\section{Références bibliographiques}

Chian L. and Wrlgram G., 1967. Tyrosinase inhibition its role in suntanning and albinism. Science, 155, 198-200.

Flawn P.C. and Wilde P.F. (a), 1970. Isolation and partial characterisation of the natural inhibitor of dopa autoxidation in skin. J. Invest. Derm., 55, 153-158.

Flawn P.C. and Wilde P.F. (b), 1970. A study of the mechanism of action and role of a natural inhibitor of dopa autoxidation isolated from Guinea pig skin. J. Invest. Derm., 55, 159-163.

Foster M., 1965. Mammalian pigment genetics. Adv. Genetics., 13, 311-339.

Gaudin H. and Fellman G., 1972. Tyrosinase in albino Rat skin. Biochim. Biophys. Acta, 141, 64-70.

Hearing V.J., 1973. Tyrosinase activity in subcellular fractions of black and albino Mice. Nature, London, 246, 81-83.

Holstein T.J., BurnetT J.B. and Quevedo W.C., 1967. Genetic regulation of multiple forms of tyrosinase in Mice. Action of $A$ and B loci. Proc. Soc. exp. Biol. and Med., 126, 415-418.

Holstein T.J., Quevedo W.C. and BurnetT J.B., 1971. Multiple forms of tyrosinase in Rodents and Lagomorphs with special reference to their genetic control in Mice. J. exp. Zool., 177, 173-183.

Kugelman T.P. and Van ScotT E.J., 1961. Tyrosinase activity in melanocytes of human albinos. J. Invest. Derm., 37, 73-76.

Pomerantz S.H., 1964. Tyrosine hydroxylation catalized by mammalian tyrosinase. An improved method of assay. Biochim. Biophys. Res. Communication, 16, 188-194.

Pomerantz S.H. and LI J.P.C., 1974. Tyrosinase in the skin of albino Hamster and Mice. Nature, London, 252, 141-143.

Prota G. and Searle A.G., 1978. Biochemical sites of gene action for melanogenesis in Mammals. Ann. Genet. Select. Anim., 10, 1-8.

Robinson R., 1958. Genetics of the Rabbit. Bibligr. Genet., 17, 229-558.

Satoh G. and Mishima Y., 1967. Tyrosinase inhibitor in Fortner amelanotic and melanotic melanoma. J. Invest. Derm., 48, 301-312.

Searle A.G., 1967. Comparative Genetics of Coat colour in Mammals. Logos Academic Press, 65.

Seiji M., Fukuzawa N., Miyazaki N., Akiba H. and Kato T., 1973. Regulatory mechanism of tyrosinase activity in melanocytes. Yale J. Biol. Med., 46, 508-515.

Seiji M., Yoshida T., Itakura H. and Irimajiri T., 1969. Inhibition of melanin formation by sulfhydryl compounds. J. Invest. Derm., 52, 280-284.

Seiji M., Yoshida T., Sakurane T. and Ogura R., 1967. Sulfhydryl content in subcellular fractions of Mouse melanoma. J. Invest. Derm., 48, 230-236.

Voulot C. et Laviolette P., 1976. Les tyrosinases des parties pigmentées de l'œil chez quatre espèces de Rongeurs. C.R. Acad. Sc. Paris, 283, 79-81.

Voulot C. et ORtonne J.P., 1979. Electrophoretic study of tyrosinase from Mammalian Pigmented Ocular Tissues, Pigment Cell., Karger, 4, 226-233.

Witkop G.J., Hill C.W., Desnick J., Thies J.K., Thorn H.L., Jenkins M. and White G.J., 1973. Ophtalmologic, Biochemical, Platelet and ultrastructural defects in the various types of oculocutaneous albinism. J. Invest. Derm., 60, 443-456. 